\title{
The wavelet transform as a tool for the characterization of the vascular response in the human lower limb
}

\author{
A transformada de wavelet como uma ferramenta para a caracterização da resposta vascular \\ no membro inferior humano
}

\author{
Henrique Silva ${ }^{1,2 *}$, Hugo Ferreira ${ }^{3}$, Julia Bujan ${ }^{4}$ and L Monteiro Rodrigues ${ }^{1,2}$ \\ ${ }^{1}$ CBIOS - Universidade Lusófona's Research Center for Biosciences and Health Technologies (UDE), Campo Grande 376, \\ 1749-024, Lisboa, Portugal \\ ${ }^{2}$ Pharmacol. Sc Depart - Universidade de Lisboa, School of Pharmacy, Lisboa, Portugal \\ ${ }^{3}$ Instituto de Biofísica e Engenharia Biomédica, Faculdade de Ciências da Universidade de Lisboa, Lisboa, Portugal \\ ${ }^{4}$ Faculty of Medicine, Alcalá de Henares, Madrid, Spain \\ Email: hnnsilva@hotmail.com
}

*This project is supported by the grant PADDIC 2013-2014, from ALIES-COFAC and part of the PhD Program in Health Sciences from U Alcalá and U Lusófona.

*Este projeto é apoiado pela bolsa PADDIC 2012-2013, da ALIES-COFAC e faz parte do Programa de Doutoramento em Ciências da Saúde da U Alcalá e U Lusófona:

\begin{abstract}
In recent years, cutaneous microcirculation gained considerable interest for the assessment of microcirculation function and dysfunction mechanisms. Laser Doppler Flowmetry (LDF) is a noninvasive and widely employed technology to study microcirculation. It provides a complex signal that may be decomposed by wavelet analysis into several components at characteristic frequency ranges. These are related to the heart $(0.6 \mathrm{~Hz}$ to $2 \mathrm{~Hz})$, respiration $(0.15 \mathrm{~Hz}$ to $0.6 \mathrm{~Hz})$, myogenic activity in the vessel wall $(0.052 \mathrm{~Hz}$ to $0.15 \mathrm{~Hz})$, sympathetic activity $(0.021 \mathrm{~Hz}$ to $0.052 \mathrm{~Hz})$ and endothelial (nitric oxide-mediated) activity $(0.0095 \mathrm{~Hz}$ to $0.021 \mathrm{~Hz})$. A group of 30 healthy $(22.3 \pm 3.1)$ years old subjects, giving previously informed consent, tested two perfusion-restricting protocols which allowed us to study the circulatory response in the lower limb: (1) passive leg elevation from the supine position for 10 minutes, (2) inflated cuff occlusion at the ankle level for 3 minutes from the sitting position. The LDF signal was partitioned using a Morlet wavelet model. Data analysis revealed significantly lower components' amplitudes for both protocols during provocation, with consistently lower values for protocol 1 in relation to protocol 2. Significant gender differences were found for protocol 1 but not for protocol 2. These results demonstrate the usefulness of the wavelet analysis to assess each LDF signal component as part of the physiological response to the lower limb microcirculation regulation.
\end{abstract}

Keywords: Microcirculation, Laser Doppler Flowmetry, wavelet transform

\begin{abstract}
Resumo
Nos últimos anos, a microcirculação cutânea ganhou considerável interesse para a avaliação dos mecanismos de função e disfunção da microcirculação. A FLD é uma tecnologia não invasiva e amplamente utilizada para estudar a microcirculação. Fornece um sinal complexo que pode ser decomposto por análise de ôndula em vários componentes em intervalos de frequência característicos. Estas estão relacionadas com a atividade cardíaca (de $0,6 \mathrm{~Hz}$ a $2 \mathrm{~Hz}$ ), respiratória (de $0,15 \mathrm{~Hz}$ a $0,6 \mathrm{~Hz}$ ), atividade miogénica na parede do vaso (de $0,052 \mathrm{~Hz}$ a $0,15 \mathrm{~Hz}$ ), atividade simpática (de $0,021 \mathrm{~Hz}$ a $0,052 \mathrm{~Hz}$ ) e atividade endotelial (mediada por óxido nítrico) (de $0,0095 \mathrm{~Hz}$ a $0,021 \mathrm{~Hz}$ ). Um grupo de 30 indivíduos saudáveis,$(22,3 \pm 3,1)$ anos), dando previamente o seu consentimento informado, testou dois protocolos de restrição de perfusão que nos permitiu estudar a resposta circulatória no membro inferior (1) elevação passiva da perna a partir da posição supina durante 10 minutos; (2) oclusão com manga insuflável ao nível do tornozelo durante 3 minutos a partir da posição sentada. 0 sinal LDF foi decomposto usando um modelo de ôndula de Morlet. A análise dos dados revelou amplitudes das componentes significativamente mais baixas para ambos os protocolos durante a provocação, com valores consistentemente mais baixos para o protocolo 1 em relação ao protocolo 2. Diferenças significativas de género foram encontradas para o protocolo 1, mas não para o protocolo 2. Estes resultados demonstram a utilidade da análise de ôndula para avaliar cada componente do sinal de FLD, como parte da resposta fisiológica na regulação da microcirculação do membro inferior.
\end{abstract}

Palavras-chave: Microcirculação, Fluxometria por Laser Doppler, transformada de wavelet 


\section{Introduction}

In recent years, the study of cutaneous microcirculation has gained considerable interest among researchers. The skin is easily accessible ${ }^{[1]}$ and potentially representative ${ }^{[2]}$ for the evaluation of peripheral microcirculation function and dysfunction mechanisms ${ }^{[3-5]}$, in particular when noninvasive technologies such as Laser Doppler Flowmetry (LDF) are used. LDF is among the most widely employed technologies for this purpose ${ }^{[6]}$, providing a complex signal which can be decomposed into components with characteristic frequency ranges by means of spectral analysis tools, such as the fast Fourier transform, autoregressive modelling and wavelet analysis ${ }^{[7]}$. A wavelet transform allows the isolation of a given structure in the physical space and in the Fourier space. The localization property of wavelets makes the wavelet analysis capable of analyzing the non-stationary systems and detecting dynamic parameters ${ }^{[7,8]}$. The Morlet wavelet, in particular, provides good time resolution at high frequencies and the best frequency resolution for low-frequency components ${ }^{[9]}$. The mentioned components are related to several perfusion-regulating activities - heart $(0.6 \mathrm{~Hz}$ to 2 $\mathrm{Hz})$, respiration $(0.15 \mathrm{~Hz}$ to $0.6 \mathrm{~Hz})$, myogenic activity in the vessel wall $(0.052 \mathrm{~Hz}$ to $0.15 \mathrm{~Hz})$, sympathetic activity $(0.021 \mathrm{~Hz}$ to $0.052 \mathrm{~Hz})$ and endothelial (nitric oxide-mediated) activity $(0.0095 \mathrm{~Hz} \text { to } 0.021 \mathrm{~Hz})^{[10,11]}$. A common approach for the assessment of the cutaneous microcirculatory status is to perform provocation tests which induce local or systemic changes in the skin blood flow (e.g. postural changes, arterial occlusion and oxygen breathing) ${ }^{[12,13]}$. Our aim was to explore these evoked changes in the LDF signal components in the lower limb skin microcirculation by applying two different perfusion restriction protocols.

\section{Materials and Methods}

A group of 30 healthy subjects, both genders (15 male, 15 female), aged between 18 and $26(22.3 \pm 3.1)$ years old, giving previously informed consent, was selected following predefined inclusion and non-inclusion criteria. All procedures complied with the ethical standards for human research by the Declaration of Helsinki and subsequent amendments ${ }^{[14]}$. Local blood flow, expressed in arbitrary units (AU) was assessed by LDF (Periflux PF 5010 system, Perimed, Sweden), whose probe was applied on the inferior side of the second toe.

\section{Introdução}

Nos últimos anos, o estudo da microcirculação cutânea ganhou interesse entre os investigadores. A pele é facilmente acessível ${ }^{[1]}$ e potencialmente representativa ${ }^{[2]}$ para a avaliação dos mecanismos de função e disfunção da microcirculação periférica ${ }^{[3-5]} \mathrm{em}$ particular quando são usadas tecnologias não invasivas, como a Fluxometria por Laser Doppler (FLD). A FLD está entre as tecnologias mais amplamente utilizadas para este propósito ${ }^{[6]}$, fornecendo um sinal complexo que pode ser decomposto em componentes com intervalos de frequência característicos, por meio de ferramentas de análise espectrais, tais como a transformada rápida de Fourier, a modelação autorregressiva e a análise de ôndula ${ }^{[7]}$. A transformada de ôndula permite o isolamento de uma dada estrutura no espaço físico e no espaço de Fourier. A propriedade de localização das ôndulas torna a análise de ôndula capaz de analisar os sistemas não-estacionários e de detetar os parâmetros dinâmicos ${ }^{[7,8]}$. A wavelet Morlet, em particular, oferece uma boa resolução temporal em altas frequências e a melhor resolução de frequência para os componentes de baixa frequência ${ }^{[9]}$. As componentes mencionadas estão relacionados com várias atividades de regulação da perfusão - atividade cardíaca (de $0,6 \mathrm{~Hz}$ a $2 \mathrm{~Hz}$ ), respiratória (de $0,15 \mathrm{~Hz}$ a $0,6 \mathrm{~Hz}$ ), atividade miogénica na parede do vaso (de $0,052 \mathrm{~Hz}$ a $0,15 \mathrm{~Hz}$ ), atividade simpática (de $0,021 \mathrm{~Hz}$ a $0,052 \mathrm{~Hz}$ ) e atividade endotelial (mediada pelo óxido nítrico) (de $0,0095 \mathrm{~Hz}$ a 0.021 $\mathrm{Hz})^{[10,11]}$. Uma abordagem comum para a avaliação do status da microcirculação cutânea é a realização de testes de provocação que induzam alterações locais ou sistémicas no fluxo sanguíneo cutâneo (ex: alterações posturais, oclusão arterial e inalação de oxigénio) ${ }^{[12,13]}$. O nosso objetivo foi explorar essas alterações nas componentes do sinal de FDL na microcirculação do membro inferior provocadas pela aplicação de dois protocolos de restrição de perfusão diferentes.

\section{Materiais e Métodos}

Um grupo de 30 indivíduos saudáveis, de ambos os sexos (15 do sexo masculino, 15 do sexo feminino) com idades entre 18 e $26(22,3 \pm 3,1)$ anos, dando previamente o seu consentimento informado, foi escolhido após observação de critérios de inclusão e de não-inclusão. Todos os procedimentos cumpriram com as normas éticas para pesquisa com seres humanos descritos na Declaração de Helsínquia e emendas subsequentes ${ }^{[14]}$. O fluxo sanguíneo local, expresso em unidades arbitrárias (UA) foi avaliado por FLD (Sistema Periflux 
Volunteers were acclimatized for 30 minutes, sitting upright with their feet exposed. Two perfusion-restricting protocols were applied. Data recording involved three phases - resting (phase I), provocation (phase II) and recovery (phase III):

\section{Protocol 1: leg elevation from the supine position}

The subject remained in a supine position for $10 \mathrm{~min}-$ utes with both legs at the heart level. Then, the measurement leg was passively raised at a 45 degrees angle for 10 minutes. After this period, the initial position is resumed for another 10 minutes.

\section{Protocol 2: occlusion with a pressure cuff}

The subject remained seated in the upright position with an empty cuff (PF 5051 Pressure Accessory Kit) placed approximately $1 \mathrm{~cm}$ above the ankle for 10 minutes. Then, the cuff is manually inflated to $200 \mathrm{mmHg}$ for 3 minutes. After this period the cuff is rapidly deflated allowing recovery for another 10 minutes.

The LDF signal, sampled at $32 \mathrm{~Hz}$, was partitioned using a Morlet wavelet transform (MATLAB). The LDF signal component amplitudes were compared for each phase of the protocol by the Wilcoxon matched-pairs signed-rank test. Gender and protocol procedure contributions were also compared with the Mann-Whitney U Test for independent samples (SPSS 21.0). A $95 \%$ confidence interval was adopted.

\section{Results and Discussion}

Spectral analysis revealed highest amplitude values for the sympathetic and endothelial components in both protocols, indicating that LDF signal was predominantly from sympathetic and endothelial origin (Figure 2).

The results from protocol 1 show that all components' amplitudes are significantly reduced in the leg elevation period. During recovery, only the cardiac and endothelial components failed to return to baseline, which suggests that the leg elevation had a prolonged effect on the cardiac activity and endothelial nitric oxide synthesis (Table 1).

The results from protocol 2 show that all activities are significantly reduced during the occlusion period, except for the sympathetic component. In the recovery period, all components return to baseline, meaning that under the present experimental conditions, a full perfu-
PF 5010, Perimed, Suécia), cuja sonda foi aplicada no lado inferior do segundo dedo do pé. Os voluntários aclimatizaram durante 30 minutos, sentados com os pés expostos. Foram aplicados dois protocolos de restrição de perfusão e o registo envolveu três fases - repouso (fase I), provocação (fase II) e recuperação (fase III):

\section{Protocolo 1: elevação da perna a partir da posição supina}

O voluntário permanece em posição supina por 10 minutos com ambas as pernas à altura do coração. Em seguida, a perna sob avaliação é elevada passivamente a um ângulo de 45 graus durante 10 minutos. Após este período a posição inicial é retomada por mais 10 minutos.

\section{Protocolo 2: oclusão com uma manga de pressão}

$\mathrm{O}$ voluntário permanece sentado com uma manga de pressão vazia (PF 5051 Pressure Accessory Kit) colocada aproximadamente $1 \mathrm{~cm}$ acima do tornozelo por 10 minutos. Em seguida, a manga é insuflada manualmente a $200 \mathrm{mmHg}$ durante 3 minutos. Após este período, a manga é rapidamente desinsuflada, permitindo a recuperação por mais 10 minutos.

$\mathrm{O}$ sinal de LDF, adquirido a uma frequência de $32 \mathrm{~Hz}$, foi decomposto usando uma transformada ôndula de Morlet (MATLAB). As amplitudes das componentes do sinal de FDL foram comparados para cada fase de cada protocolo teste de Wilcoxon para amostras emparelhadas. As contribuições do género e protocolo foram também comparadas com o teste U de Mann-Whitney para amostras independentes (SPSS 21.0). Foi adotado um intervalo de confiança de $95 \%$.

\section{Resultados e Discussão}

A análise espectral revelou os maiores valores de amplitude para as componentes simpática e endotelial em ambos os protocolos, o que significa que o sinal de FDL foi predominantemente de origem simpática e endotelial (Figura 2).

Os resultados para o protocolo 1 mostram que as amplitudes de todas as componentes são significativamente reduzidas durante a elevação passiva da perna. Durante a recuperação, apenas as componentes cardíaca e endotelial não retornaram à linha de base, o que sugere que a elevação da perna teve um efeito prolongado sobre a atividade cardíaca e síntese endotelial de óxido nítrico (Tabela 1).

Os resultados do protocolo 2 mostram que todas as atividades são significativamente reduzidas durante o período de oclusão, exceto para a componente simpática. No período de recuperação, todas as componentes retornaram à linha de base, o que significa que nas presentes condições experimentais ocorreu 
sion recovery takes place (Table 1 ). These results agree with previously published studies ${ }^{[15]}$.

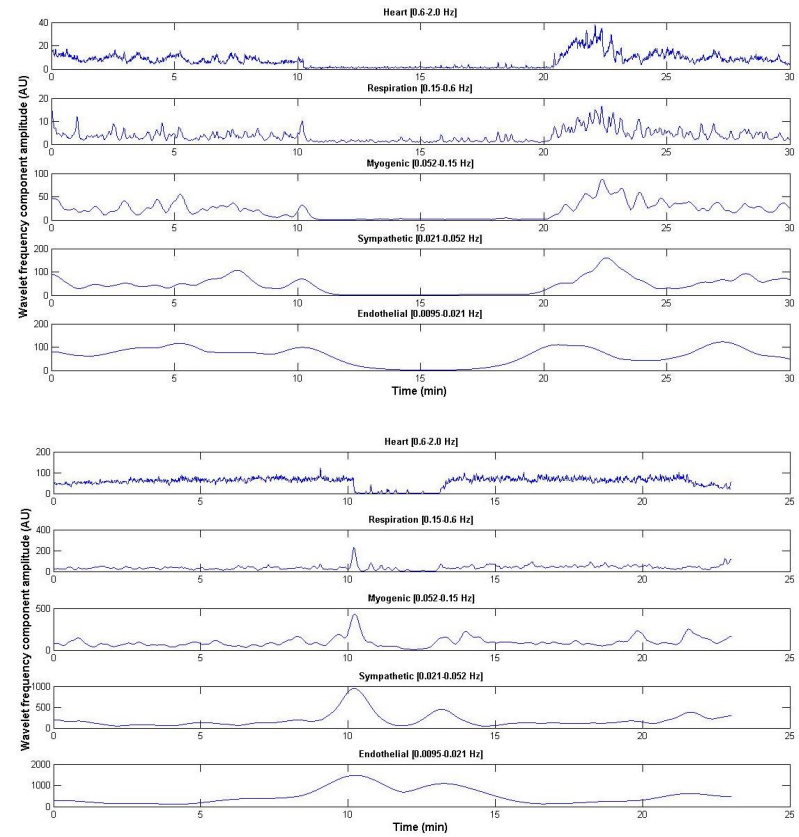

Figure 2 / Figura 2 - Amplitude over time of each frequency component: heart (from $0.6 \mathrm{~Hz}$ to $2 \mathrm{~Hz}$ ), respiration (from $0.15 \mathrm{~Hz}$ to $0.6 \mathrm{~Hz}$ ), myogenic activity in the vessel wall (from $0.052 \mathrm{~Hz}$ to $0.15 \mathrm{~Hz}$ ), sympathetic activity (from $0.021 \mathrm{~Hz}$ to $0.052 \mathrm{~Hz}$ ) and endothelial metabolic activity (from 0.0095 $\mathrm{Hz}$ to $0.021 \mathrm{~Hz}$ ) obtained for protocol 1 (top) and protocol 2 (bottom). Data is from a representative subject.

Amplitude ao longo do tempo para cada componente de frequência: cardíaca (de $0,6 \mathrm{~Hz}$ a $2 \mathrm{~Hz}$ ), respiratória (de $0,15 \mathrm{~Hz}$ a $0,6 \mathrm{~Hz}$ ), atividade miogénica na parede do vaso (de $0,052 \mathrm{HZ}$ a $0,15 \mathrm{~Hz}$ ), atividade simpática (de $0,021 \mathrm{~Hz}$ a $0,052 \mathrm{~Hz}$ ) e atividade endotelial (de $0,0095 \mathrm{~Hz}$ a $0,021 \mathrm{~Hz}$ ) obtido para o protocolo 1 (em cima) e protocolo

In protocol 1, all the components' amplitudes were significantly lower than those in protocol 2 in all phases, likely related to the perfusion differences determined by the body positioning. In the supine position, blood is redistributed along the body, lowering perfusion in the lower limb, while in the sitting position, gravity promotes higher blood content in the lower limb (Figure 1). uma recuperação completa de perfusão (Tabela 1). Estes resultados suportam outros publicados anteriormente ${ }^{[15]}$.

Table 1 / Tabela 1. Mean values and standard deviation of each LDF component for the last 3 minutes phase II and the last 5 minutes of phase III during the applied protocols. Statistical comparison for the last 5 minutes of phase I is also shown (AU - arbitrary units; NS - non significant; $\mathrm{p}<.05$ ).

Valores médios (e desvio-padrão) de cada componente do sinal de FLD para os últimos 3 minutos da fase II e últimos 5 minutos da fase III dos protocolos aplicados. A comparação estatística para os últimos 5 minutos de fase I é também incluída na tabela (UA - unidades arbitrárias; NS - não significativo; $\mathrm{p}<.05$ ).

\begin{tabular}{|c|c|c|c|c|}
\hline \multirow{2}{*}{\multicolumn{2}{|c|}{ Component / Componente }} & \multicolumn{3}{|c|}{ Wavelet Amplitude (AU) / Amplitude de ôndula (UA) } \\
\hline & & Phase I/ Fase I & Phase II / Fase II & Phase III / Fase III \\
\hline \multirow{10}{*}{$\begin{array}{l}\text { Protocol } 1 / \\
\text { Protocolo } 1\end{array}$} & \multirow{2}{*}{$\begin{array}{c}\text { Heart I } \\
\text { Cardíaca }\end{array}$} & $4.0 \pm 2.9$ & $2.9 \pm 2.3$ & $3.7 \pm 3.2$ \\
\hline & & - & $p<.001$ & .037 \\
\hline & \multirow{2}{*}{$\begin{array}{l}\text { Respiration / } \\
\text { Respiratória }\end{array}$} & $3.4 \pm 3.5$ & $2.0 \pm 1.1$ & $3.0 \pm 2.2$ \\
\hline & & - & .001 & NS \\
\hline & \multirow{2}{*}{$\begin{array}{l}\text { Myogenic / } \\
\text { Miogénica }\end{array}$} & $15.6 \pm 16.3$ & $4.9 \pm 5.0$ & $17.5 \pm 21.2$ \\
\hline & & - & $\mathrm{p}<.001$ & NS \\
\hline & \multirow{2}{*}{$\begin{array}{l}\text { Sympathetic } \\
\text { / Simpática }\end{array}$} & $35.4 \pm 28.3$ & $15.1 \pm 14.4$ & $33.6 \pm 30.3$ \\
\hline & & - & $p<.001$ & NS \\
\hline & \multirow{2}{*}{$\begin{array}{c}\text { Endothelial / } \\
\text { Endotelial }\end{array}$} & $51.8 \pm 45.0$ & $41.1 \pm 38.3$ & $46.1 \pm 46.5$ \\
\hline & & - & .009 & .022 \\
\hline \multirow{10}{*}{$\begin{array}{l}\text { Protocol } 21 \\
\text { Protocolo } 2\end{array}$} & \multirow{2}{*}{$\begin{array}{c}\text { Heart I } \\
\text { Cardíaca }\end{array}$} & $11.6 \pm 13.5$ & $3.3 \pm 3.3$ & $10.8 \pm 12.5$ \\
\hline & & - & $p<.001$ & NS \\
\hline & \multirow{2}{*}{$\begin{array}{l}\text { Respiration / } \\
\text { Respiratória }\end{array}$} & $18.5 \pm 23.6$ & $7.3 \pm 5.7$ & $16.9 \pm 20.7$ \\
\hline & & - & .001 & NS \\
\hline & \multirow{2}{*}{$\begin{array}{l}\text { Myogenic / } \\
\text { Miogénica }\end{array}$} & $61.0 \pm 52.1$ & $32.6 \pm 26.2$ & $57.1 \pm 41.8$ \\
\hline & & - & $p<.001$ & NS \\
\hline & \multirow{2}{*}{$\begin{array}{l}\text { Sympathetic } \\
\text { / Simpática }\end{array}$} & $128.5 \pm 108.8$ & $122.9 \pm 102.7$ & $109.7 \pm 86.4$ \\
\hline & & - & NS & NS \\
\hline & \multirow{2}{*}{$\begin{array}{c}\text { Endothelial / } \\
\text { Endotelial }\end{array}$} & $226.9 \pm 233.0$ & $372.1 \pm 297.3$ & $195.0 \pm 153.7$ \\
\hline & & - & $p<.001$ & NS \\
\hline
\end{tabular}

No protocolo 1 todas as amplitudes das componentes foram significativamente menores que no protocolo 2 , em todas as fases, o que está provavelmente relacionado com as diferenças de perfusão determinadas pelo posicionamento do corpo. Em supina, o sangue é redistribuído ao longo do corpo, reduzindo a perfusão no membro inferior, enquanto que na posição sentada a gravidade promove um maior conteúdo de sangue no membro inferior (Figura 1).
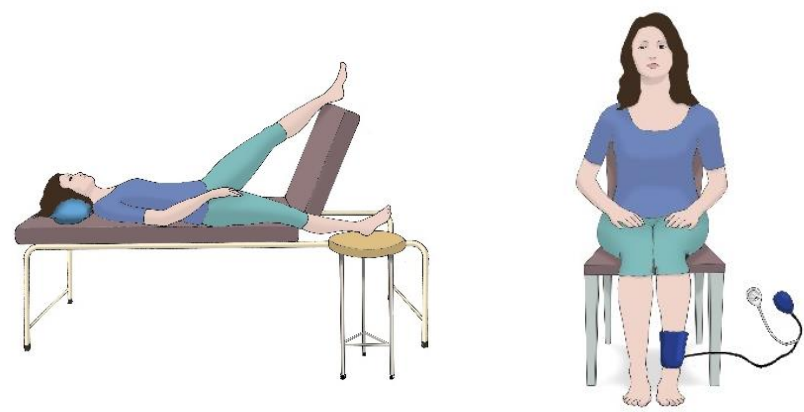

Figure 1 / Figura 1: Illustration of the experimental maneuvers adopted in protocols 1 (left) and 2 (right). Ilustração das manobras experimentais adotadas nos protocolos 1 (à esquerda) e 2 (à direita). 
The cardiac activity during the provocation phase was the exception, as no statistical significant differences between protocols could be demonstrated (Table 2).

The components' amplitudes were generally higher in males in protocol 1, except for the recovery phase of the cardiac component and the provocation phase of the respiratory component (Table 3 ).

No statistical differences between genders were detected in protocol 2, suggesting that gender is not a determinant of blood perfusion regulation in post-occlusion hyperemia (Table 3 ).

Table 2 / Tabela 2. Statistical comparison of each phase of each LDF signal component between protocols (NS - non significant; $\mathrm{p}<.05$ ).

Comparação estatística de cada fase de cada componente do sinal de FLD entre os protocolos (NS - não significativo; $\mathrm{p}<.05)$.

\begin{tabular}{|c|c|c|c|}
\hline Component / Componente & $\begin{array}{c}\text { Phase I/ } \\
\text { Fase I }\end{array}$ & $\begin{array}{c}\text { Phase II I } \\
\text { Fase II }\end{array}$ & $\begin{array}{c}\text { Phase III } \\
\text { I Fase III }\end{array}$ \\
\hline Heart / Cardíaca & $<.001$ & NS & $<.001$ \\
\hline Respiration / Respiratória & $<.001$ & $<.001$ & $<.001$ \\
\hline Myogenic / Miogénica & $<.001$ & $<.001$ & $<.001$ \\
\hline Sympathetic / Simpática & $<.001$ & $<.001$ & $<.001$ \\
\hline Endothelial / Endotelial & $<.001$ & $<.001$ & $<.001$ \\
\hline
\end{tabular}

\section{Conclusions}

The present results confirm the wavelet transform's utility in assessing the individual contribution of each LDF signal component to different physiological responses involved in the regulation of lower limb microcirculation.

\section{Acknowledgements}

The authors would like to express their thanks to Joana Antunes for the collaboration and assistance and to volunteers for their participation.

\section{Conflict of Interests}

The authors declare that there are no financial and/or personal relationships that could be viewed as presenting a potential conflict of interests.
A atividade cardíaca durante a fase de provocação foi a exceção, já que diferenças estatísticas significativas entre os protocolos não puderam ser demonstradas (Tabela 2).

As amplitudes das componentes foram geralmente maiores no género masculino no protocolo 1 , com exceção da fase de recuperação da componente cardíaca e a fase de provocação da componente respiratória (Tabela 3).

Não foram detetadas diferenças estatísticas entre géneros no protocolo 2 , sugerindo que o género não é um fator determinante da regulação da perfusão sanguínea na hiperémia pós-oclusão (Tabela 3).

Table 3 / Tabela 3. Statistical comparison of each phase of each LDF signal component between genders for each protocol (NS - non significant; $\mathrm{p}<.05$ ).

Comparação estatística de cada fase de cada componente do sinal de FLD entre géneros para cada protocolo (NS - não significativo; $\mathrm{p}<.05$ ).

\begin{tabular}{|c|c|c|c|c|c|c|}
\hline \multirow{2}{*}{ Component / Componente } & \multicolumn{3}{|c|}{ Protocol 1 / Protocolo 1 } & \multicolumn{3}{c|}{ Protocol 2 / Protocolo 2 } \\
\cline { 2 - 7 } & $\begin{array}{c}\text { Phase I/ } \\
\text { Fase I }\end{array}$ & $\begin{array}{c}\text { Phase II I } \\
\text { Fase II }\end{array}$ & $\begin{array}{c}\text { Phase III } \\
\text { /Fase III }\end{array}$ & $\begin{array}{c}\text { Phase I/ } \\
\text { Fase I }\end{array}$ & $\begin{array}{c}\text { Phase II I } \\
\text { Fase II }\end{array}$ & $\begin{array}{c}\text { Phase III } \\
\text { /Fase III }\end{array}$ \\
\hline Heart / Cardiaca & .013 & .041 & NS & NS & NS & NS \\
\hline Respiration / Respiratória & .010 & NS & .026 & NS & NS & NS \\
\hline Myogenic / Miogénica & .019 & .089 & .005 & NS & NS & NS \\
\hline Sympathetic / Simpática & .016 & .011 & .016 & NS & NS & NS \\
\hline Endothelial / Endotelial & .067 & .037 & .026 & NS & NS & NS \\
\hline
\end{tabular}

\section{Conclusões}

Os resultados deste estudo confirmam a utilidade da transformada de ôndula na avaliação da contribuição individual de cada componente do sinal de FLD para diferentes respostas fisiológicas envolvidas na regulação da microcirculação do membro inferior.

\section{Agradecimentos}

Os autores gostariam de expressar seus agradecimentos a Joana Antunes pela colaboração e assistência e aos voluntários pela sua participação.

\section{Conflito de Interesses}

Os autores declaram não existir qualquer relação pessoal ou financeira que possa ser entendida como representando um potencial conflito de interesses. 


\section{References / Referências}

1. Lenasi H. Assessment of human skin microcirculation and its endothelial function using laser Doppler flowmetry. In: Erondu OF (ed.) Medical imaging. Rijeka: InTech, 2011, pp. 271-296.

2. Holowatz LA, Kenney WL. Up-regulation of arginase activity contributes to attenuated reflex cutaneous vasodilatation in hypertensive humans. J Physiol 2007; 581:863-872.

3. Abularrage CJ, Sidawy AN, Aidinian G, Singh N, Weiswasser JM, Arora S. Evaluation of the microcirculation in vascular disease. J Vasc Surg 2005; 42:574-581.

4. Izjerman RG, de Jongh RT, Beijk MA, van Weissenbruch MM, Delemarre-van de Waal HA, Serne EH, Stehouwer CD. Individuals at increased coronary heart disease risk are characterized by an impaired microvascular function in skin. Eur J Clin Invest 2003; 33:536-542.

5. Stewart J, Kohen A, Brouder D, Rahim F, Adler S, Garrick R, Goligorsky MS. Noninvasive interrogation of microvasculature for signs of endothelial dysfunction in patients with chronic renal failure. Am J Physiol Heart Circ Physiol 2004; 287:H2687-E2696.
6. Roustit M, Cracowski JL. Non-invasive assessment of skin microvascular function in humans: an insight into methods. Microcirculation. 2012;19(1):47-64. doi: 10.1111/j.1549-8719.2011.00129.x.

7. Holshneider M. Wavelets: an analysis tool. Oxford: Clarendon Press, 1995.

8. Sosnovtseva OV, Pavlov AN, Mosekilde E, Holstein-Rathlou N-H, Marsh DJ. Doublewavelet approach to study frequency and amplitude modulation in renal autoregulation. Physical Review E 2004; 70:031915.

9. Smirnova E, Podtaev S, Mizeva I, Loran E. Assessment of endothelial dysfunction in patients with impaired glucose tolerance during a cold pressor test. Diab Vasc Dis Res. 2013;10(6):489-97. doi: $10.1177 / 1479164113494881$.

10. Oberg PA. Laser-Doppler flowmetry. Critical Reviews in Biomedical Engineering 1990; 18:125-163.

11. Landsverk SV, Kvandal P, Bernjak A, Stefanovska A, Kirkeboen KA. The effects of general anesthesia on human skin microcirculation evaluated by wavelet transform. Anesthesia \& Analgesia 2007; 105:1012-1019.
12. Silva H, Ferreira H, Tavares L, Bujan J, Rodrigues LM. Exploring in vivo models to characterize peripheral microcirculation - a pilot study. Biomed Biopharm Res. 2013; (10)1:65-72.

13. Silva H, Ferreira H, Bujan J, Rodrigues LM. Exploring the oxygen challenge test as a microcirculation evaluation model. Biomed Biopharm Res. 2013; (10)2:209-215.

14. World Medical Association. JAMA. 2013; 310(20):2191-4. World Medical Association Declaration of Helsinki: ethical principles for medical research involving human subjects. doi: 10.1001/jama.2013.281053.

15. Ferreira HA, Fernandes CD, Pinto PC, Rodrigues LM. Use of Wavelet Transforms and Detrended Fluctuation Analysis in the evaluation of human skin microcirculation. Biomedical and Biopharmaceutical Research 2011; 1(9): 79-86. 